\title{
Cavity-Backed Cylindrical Wraparound Antennas
}

\author{
O. M. C. Pereira-Filho' ${ }^{1}$, T. B. Ventura ${ }^{2}$, C. G. Rego ${ }^{3}$, \\ A. F. Tinoco-S. ${ }^{4}$, and J. C. da S. Lacava ${ }^{4}$ \\ ${ }^{1}$ Federal University of Pernambuco, \\ ${ }^{2}$ UNA College, \\ ${ }^{3}$ Federal University of Minas Gerais, \\ ${ }^{4}$ Technological Institute of Aeronautics
}

Brazil

\section{Introduction}

One of the most important advantages of microstrip antennas is their ability to conform to various surfaces, accordingly to mechanical or aerodynamical needs. Cavity-backed cylindrical wraparound antennas are microstrip-ring radiators that are embedded in a circular cylinder, like a missile for example. The purpose of this chapter is to present a detailed and accurate analysis of such antennas that fully takes into account its geometry, as well as overcomes some difficulties encountered for cylindrical microstrip antennas.

Most usually cylindrical microstrip antennas refer to metallic patches printed onto dielectric-coated infinite cylinders. Various methods have been applied to analyze such antennas (Wong,1999), as cavity method, electric surface current method, method of moments, and hybrid methods. The cavity method (Krowne,1983; Luk et al.,1989; Lumini,1991; Heckler et al.,2003; Yang \& Ruan,1993; Wong \& Ke,1993) is based on the fact that the electromagnetic fields are concentrated between the patch and the cylinder, and can be approximated by a modal expansion within this cavity. The dielectric layer external to the cavity is usually disregarded and equivalent magnetic surface currents are used as radiation sources. Although limited to thin dielectrics the cavity method provides valuable insight information about the field distribution. The dielectric effect may be included by the Green's functions as in (Fonseca \& Giarola,1983). The electric surface current method (Ashkenazy et al.,1985) uses an approximation of the electric surface current on the antenna and the Green's functions to calculate the electrical characteristics of the antenna. It presents more accurate results than cavity method as the dielectric layer is taken into account through the Green's functions. Full-wave analysis have also been extensively employed to analyze a variety of microstrip antennas through the method of moments (Harrington,1968). This procedure expands the surface current distribution on the antenna in a set of functions known as basis-functions. The fields are obtained using Green's functions, and the expansion coefficients are determined by imposing the boundary conditions. The complexity of such method depends on the choice of these functions. It was used in (Ali et al.,1989) and (Habashy et al.,1990) to determine the resonance frequencies, radiation patterns and input impedances of rectangular and wraparound antennas printed on a single dielectric layer over a cylindrical conductor, when fed by a coaxial cable. The effect of parasitic rectangular patches are analyzed in (Tam et al.,1995) and cylindrical arrays of rectangular patches were 
analyzed in (Silva et al.,1991). Superstrate-loaded cylindrical microstrip antennas were also analyzed. In (Silva et al.,1991b) the rectangular patch was placed between two dielectric layers over a cylindrical conductor, and fed by a transmission line. The superstrate effect on the resonance frequency of rectangular patches was described in (Wong et al.,1993b), and on the input impedance of a probe-fed rectangular patch was shown in (Ke \& Wong,1994). Different configurations of arrays of cylindrical-rectangular patches in a multilayer circular cylindrical structure were shown in (Raffaelli et al.,2005). Mutual impedance of superstrate-loaded wraparound antennas was presented in (Silva \& Lacava,1995). A hybrid solution combining finite-difference time-domain and Green's functions was used in (Mang \& Xiaowen,2004) to obtain the input impedance and radiation pattern of probe-fed multilayered cylindrical-rectangular antennas.

Many practical situations require the use of embedded antennas for which the dielectric-coated infinite cylinder is no longer an accurate model. These include cavity-backed rectangular antennas (Kempel \& Volakis,1995) analyzed by hybrid finite element-boundary integral method, and flush-mounted cylindrical-rectangular microstrip antennas (Pereira Filho,2009) by method of moments. These geometries avoid the surface waves at the infinite dielectric layers, diminishing the coupling between array elements, and increasing the antenna efficiency.

The purpose of this chapter is to present a detailed analysis of a cavity-backed wraparound cylindrical antennas, using equivalence principle and method of moments. A cavity is built within a cylindrical perfect electric conductor, and filled with a dielectric. A wraparound patch is printed onto the dielectric interface. The antenna is fed by a parallel network of equally spaced coaxial cables along a circumference, allowing the antenna to radiate an almost omni-directional pattern. The procedure is based on equivalence principle to split the region in two, a cylindrical cavity and an external region, with equivalent magnetic surface currents in the interface. Method of moments is applied to determine the equivalent currents, using full-domain basis-functions in $\phi$-direction and sub-domain basis-functions in $z$-direction.

Method of moments analyses of cylindrical microstrip antennas are known to present limitations when dealing with large cylinders. This is due to numerical problems in evaluating Bessel functions of large complex arguments, that arise from the wave equation solution in cylindrical coordinates. An analytical procedure is presented that mitigate this problem and allows the analysis to be performed for larger cylinders. The procedure proposed here starts from writing the Green's function in an appropriate form, where the Bessel functions appear in specific combinations which are evaluated differently according to the argument range. For very small arguments analytical expressions of such combinations are obtained from analytical limits of the Bessel functions. These analytical limits of the combinations can be evaluated for arguments much smaller than the Bessel functions individually, due to opposite behavior of the Bessel functions in these combinations. Similarly, for very large arguments asymptotic expressions of Bessel functions are used to obtain analytical expressions of the combinations that can be calculated much further than single Bessel functions. Numerical routines of Bessel functions are only used for an argument range without numerical issues.

Results for radiation pattern and input impedance are compared to those from a commercial software or from other methods. The input impedance is obtained from a variational expression, based on reciprocity theorem. It includes the reaction of the fields due to the basis-functions on the feeding network, as well as the reaction of the fields due to the feeding network on itself, which leads to the self-impedance of the feed. Unlike for rectangular or spherical geometries, coaxial cables in cylindrical layers generate both transverse electric 


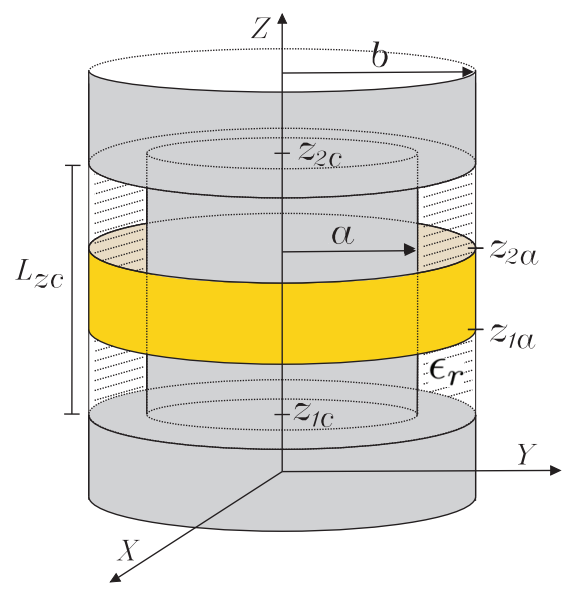

Fig. 1. Geometry of cavity-backed cylindrical wraparound antenna.

and transverse magnetic fields, and the self-impedance of the feed is not straightforward. This chapter introduces an approximation for the self-impedance of probes in cylindrically layered medium, by disregarding its radiation contribution. Comparison of the results with those obtained using commercial software validates the analysis. The effects of the cavity on radiation fields and input impedance are also presented.

\section{Application of equivalence principle}

The geometry of the cavity-backed cylindrical wraparound antenna is shown in Fig. 1. A cylindrical perfect electric conductor has radius $b$. A cavity is built within the conductor, from $z=z_{1 c}$ to $z=z_{2 c}$, with inner radius $a$, and filled with a dielectric of permeability $\mu_{d}=\mu_{o}$ and permittivity $\varepsilon_{d}=\varepsilon_{r} \varepsilon_{o}(1-j \tan \delta)$, where $\varepsilon_{r}$ and $\tan \delta$ are the relative permittivity and loss tangent of the dielectric, and $\mu_{0}$ and $\varepsilon_{0}$ are free-space permeability and permittivity, respectively. A wraparound antenna is printed onto the dielectric surface $(\rho=b)$, from $z=z_{1 a}$ to $z=z_{2 a}$. The antenna is fed by a parallel network of equally spaced $N_{p}$ coaxial cables along a circumference $\left(z=z_{f}, \rho=b\right)$ of the antenna. All conductors are assumed to be perfect, and a time-variation of $e^{j w t}$ is assumed and suppressed.

The antenna is analyzed with the aid of the equivalence principle. The original geometry shown in Fig. 1 is split into 2 regions, by adding perfect electric conductors at $\rho=b$ from $z_{1 c}$ to $z_{1 a}$ and from $z_{2 a}$ to $z_{2 c}$, and equivalent magnetic surface currents. The internal region forms a cavity from $\rho=a$ to $\rho=b$, and from $z=z_{1 c}$ to $z=z_{2 c}$. The fields within the cavity are due to the excitation probes and to the equivalent magnetic surface current $\left(\bar{M}_{s}\right)$ at $\rho=b$. The fields in the external region $(\rho>b)$ are due to the equivalent magnetic surface current $\left(-\bar{M}_{S}\right)$ at $\rho=b$ in the presence of an infinite perfect electric cylindrical conductor. The problem is solved using method of moments in spectral domain, as described below.

\subsection{Internal region}

The fields within the cavity are written as the sum of $T E_{z}$ and $T M_{z}$ components, with the aid of vector potential components $F_{z d}$ and $A_{z d}$, respectively. The boundary conditions in 
$z$-direction require that $\partial A_{z d} / \partial z=0$ and $F_{z d}=0$ at $z=z_{1 c}$ and $z=z_{2 c}$. For both cases the boundary conditions require the continuity of the potentials in $\phi$-direction. As a result the vector potentials can be expressed in a double-Fourier series already satisfying the boundary conditions in $z$ and $\phi$-directions as:

$$
\begin{aligned}
& A_{z d}(\rho, \phi, z)=\sum_{n=-\infty}^{\infty} \sum_{q=0}^{\infty} \widetilde{A}_{z d}^{e c}(\rho, n, q) e^{-j n \phi} \cos \left[\frac{q \pi}{L_{z c}}\left(z-z_{1 c}\right)\right] \\
& F_{z d}(\rho, \phi, z)=\sum_{n=-\infty}^{\infty} \sum_{q=1}^{\infty} \widetilde{F}_{z d}^{e s}(\rho, n, q) e^{-j n \phi} \sin \left[\frac{q \pi}{L_{z c}}\left(z-z_{1 c}\right)\right]
\end{aligned}
$$

where $L_{z c}=\left(z_{2 c}-z_{1 c}\right)$, the first superscript in $\tilde{A}_{z d}^{e c}$ or $\tilde{F}_{z d}^{e s}$ refers to an exponential Fourier series in $\phi$, and the second superscript refers to a sine or cosine Fourier series in $z$. And the vector potentials in spectral domain are given by:

$$
\begin{aligned}
& \widetilde{A}_{z d}^{e c}(\rho, n, q)=\frac{1}{2 \pi} \frac{\epsilon_{q}}{L_{z c}} \int_{0}^{2 \pi} \int_{z_{1 c}}^{z_{2 c}} A_{z d}(\rho, \phi, z) e^{j n \phi} \cos \left[\frac{q \pi}{L_{z c}}\left(z-z_{1 c}\right)\right] d z d \phi \\
& \widetilde{F}_{z d}^{e s}(\rho, n, q)=\frac{1}{2 \pi} \frac{2}{L_{z c}} \int_{0}^{2 \pi} \int_{z_{1 c}}^{z_{2 c}} F_{z d}(\rho, \phi, z) e^{j n \phi} \sin \left[\frac{q \pi}{L_{z c}}\left(z-z_{1 c}\right)\right] d z d \phi
\end{aligned}
$$

where $\epsilon_{q}=1$ if $q=0$, and $\epsilon_{q}=2$ if $q>0$.

In space-domain the electromagnetic fields are obtained from the vector potentials by (Harrington,1961):

$$
\begin{aligned}
E_{\rho} & =\frac{1}{j \omega \varepsilon_{d}} \frac{\partial^{2} A_{z d}}{\partial \rho \partial z}-\frac{1}{\rho} \frac{\partial F_{z d}}{\partial \phi} & H_{\rho} & =\frac{1}{j \omega \mu_{d}} \frac{\partial^{2} F_{z d}}{\partial \rho \partial z}+\frac{1}{\rho} \frac{\partial A_{z d}}{\partial \phi} \\
E_{\phi} & =\frac{1}{j \omega \varepsilon_{d} \rho} \frac{\partial^{2} A_{z d}}{\partial \phi \partial z}+\frac{\partial F_{z d}}{\partial \rho} & H_{\phi} & =\frac{1}{j \omega \mu_{d} \rho} \frac{\partial^{2} F_{z d}}{\partial \phi \partial z}-\frac{\partial A_{z d}}{\partial \rho} \\
E_{z} & =\frac{1}{j \omega \varepsilon_{d}}\left(\frac{\partial^{2}}{\partial z^{2}}+k_{d}^{2}\right) A_{z d} & H_{z} & =\frac{1}{j \omega \mu_{d}}\left(\frac{\partial^{2}}{\partial z^{2}}+k_{d}^{2}\right) F_{z d}
\end{aligned}
$$

where $k_{d}=\omega \sqrt{\varepsilon_{d} \mu_{d}}$ is the wavenumber in the dielectric, including losses. Using the double-Fourier series (1) and (2) the components of the electromagnetic fields in spectral domain are given by:

$$
\begin{aligned}
& \widetilde{E}_{\rho}^{e s}(\rho, n, q)=-\frac{1}{j \omega \varepsilon_{d}} \frac{q \pi}{L_{z c}} \frac{\partial}{\partial \rho} \widetilde{A}_{z d}^{e c}(\rho, n, q)+\frac{j n}{\rho} \widetilde{F}_{z d}^{e s}(\rho, n, q) \\
& \widetilde{E}_{\phi}^{e s}(\rho, n, q)=\frac{n}{\omega \varepsilon_{d} \rho} \frac{q \pi}{L_{z c}} \widetilde{A}_{z d}^{e c}(\rho, n, q)+\frac{\partial}{\partial \rho} \widetilde{F}_{z d}^{e s}(\rho, n, q) \\
& \widetilde{E}_{z}^{e c}(\rho, n, q)=\frac{1}{j \omega \varepsilon_{d}} k_{\rho_{d}}^{2} \widetilde{A}_{z d}^{e c}(\rho, n, q) \\
& \widetilde{H}_{\rho}^{e c}(\rho, n, q)=-\frac{j n}{\rho} \widetilde{A}_{z d}^{e c}(\rho, n, q)+\frac{1}{j \omega \mu_{d}} \frac{q \pi}{L_{z c}} \frac{\partial}{\partial \rho} \widetilde{F}_{z d}^{e s}(\rho, n, q) \\
& \widetilde{H}_{\phi}^{e c}(\rho, n, q)=-\frac{\partial}{\partial \rho} \widetilde{A}_{z d}^{e c}(\rho, n, q)-\frac{n}{\omega \mu_{d} \rho} \frac{q \pi}{L_{z c}} \widetilde{F}_{z d}^{e s}(\rho, n, q) \\
& \widetilde{H}_{z}^{e s}(\rho, n, q)=\frac{1}{j \omega \mu_{d}} k_{\rho_{d}}^{2} \widetilde{F}_{z d}^{e s}(\rho, n, q)
\end{aligned}
$$


where $k_{\rho_{d}}^{2}=k_{d}^{2}-\left(\frac{q \pi}{L_{z c}}\right)^{2}$.

The vector potentials in spectral domain $\left(\tilde{A}_{z}^{e c}(\rho, n, q)\right.$ and $\left.\tilde{F}_{z}^{e s}(\rho, n, q)\right)$ are combinations of Bessel functions of order $n$ satisfying the boundary conditions at $\rho=a$ and $\rho=b$. At the inner cylindrical conductor $(\rho=a)$ the zero tangential electric field requires that $A_{z}^{d}=0$ and $\partial F_{z}^{d} / \partial \rho=0$, and the potentials can be written as:

$$
\begin{aligned}
& \widetilde{A}_{z d}^{e c}(\rho, n, q)=c_{1}\left[J_{n}\left(k_{\rho_{d}} \rho\right) H_{n}^{(2)}\left(k_{\rho_{d}} a\right)-J_{n}\left(k_{\rho_{d}} a\right) H_{n}^{(2)}\left(k_{\rho_{d}} \rho\right)\right] \\
& \widetilde{F}_{z d}^{e s}(\rho, n, q)=c_{2}\left[J_{n}\left(k_{\rho_{d}} \rho\right) H_{n}^{(2)^{\prime}}\left(k_{\rho_{d}} a\right)-J_{n}^{\prime}\left(k_{\rho_{d}} a\right) H_{n}^{(2)}\left(k_{\rho_{d}} \rho\right)\right]
\end{aligned}
$$

where $B_{n}^{\prime}($.$) is the derivative of the Bessel function regarding to the argument. The boundary$ conditions at $\rho=b$ require that the equivalent surface magnetic current $\bar{M}_{\mathcal{S}}(\phi, z)=-\hat{n} \times$ $\bar{E}(b, \phi, z)=\hat{a}_{\rho} \times \bar{E}(b, \phi, z)$, where $\hat{n}$ is the unit vector normal to the surface $\rho=b$ pointing inwards. Consequently $M_{z}(\phi, z)=E_{\phi}(b, \phi, z)$ and $M_{\phi}(\phi, z)=-E_{z}(b, \phi, z)$. In spectral domain it reads:

$$
\begin{aligned}
& \widetilde{M}_{z}^{e s}(n, q)=\widetilde{E}_{\phi}^{e s}(b, n, q)=\frac{n}{\omega \varepsilon_{d} b} \frac{q \pi}{L_{z c}} \widetilde{A}_{z d}^{e c}(b, n, q)+\frac{\partial}{\partial \rho} \widetilde{F}_{z d}^{e s}(b, n, q) \\
& \widetilde{M}_{\phi}^{e c}(n, q)=-\widetilde{E}_{z}^{e c}(b, n, q)=-\frac{1}{j \omega \varepsilon_{d}} k_{\rho_{d}}^{2} \widetilde{A}_{z d}^{e c}(b, n, q)
\end{aligned}
$$

For a given equivalent surface magnetic current the coefficients $c_{1}$ and $c_{2}$ are determined by substituting (12) and (13) into (14) and (15). And the vector potentials can be written as:

$$
\begin{aligned}
\widetilde{A}_{z d}^{e c}(\rho, n, q) & =\widetilde{G}_{M \phi}^{A d}(\rho, n, q) \widetilde{M}_{\phi}^{e c}(n, q), \\
\widetilde{F}_{z d}^{e s}(\rho, n, q) & =\widetilde{G}_{M \phi}^{F d}(\rho, n, q) \widetilde{M}_{\phi}^{e c}(n, q)+\widetilde{G}_{M z}^{F d}(\rho, n, q) \widetilde{M}_{z}^{e s}(n, q)
\end{aligned}
$$

where $\tilde{G}_{M \phi}^{A d}(\rho, n, q)$ corresponds to the transform of the Green's function for the potential $A_{z}^{d}(\rho, \phi, z)$ due to a $\phi$-directed magnetic surface current at $\rho=b$. And similarly for the other terms, which are given by:

$$
\begin{aligned}
& \widetilde{G}_{M \phi}^{A d}(\rho, n, q)=-\frac{j \omega \varepsilon_{d}}{\left(k_{\rho_{d}}\right)^{2} \vartheta_{5}(n, q)}\left[J_{n}\left(k_{\rho_{d}} \rho\right) H_{n}^{(2)}\left(k_{\rho_{d}} a\right)-J_{n}\left(k_{\rho_{d}} a\right) H_{n}^{(2)}\left(k_{\rho_{d}} \rho\right)\right] \\
& \widetilde{G}_{M \phi}^{F d}(\rho, n, q)=\frac{j n}{b\left(k_{\rho_{d}}\right)^{3} \vartheta_{1}(n, q)} \frac{q \pi}{L_{z c}}\left[J_{n}\left(k_{\rho_{d}} \rho\right) H_{n}^{(2)^{\prime}}\left(k_{\rho_{d}} a\right)-J_{n}^{\prime}\left(k_{\rho_{d}} a\right) H_{n}^{(2)}\left(k_{\rho_{d}} \rho\right)\right] \\
& \widetilde{G}_{M z}^{F d}(\rho, n, q)=\frac{1}{\left(k_{\rho_{d}}\right) \vartheta_{1}(n, q)}\left[J_{n}\left(k_{\rho_{d}} \rho\right) H_{n}^{(2)^{\prime}}\left(k_{\rho_{d}} a\right)-J_{n}^{\prime}\left(k_{\rho_{d}} a\right) H_{n}^{(2)}\left(k_{\rho_{d}} \rho\right)\right]
\end{aligned}
$$

where:

$$
\begin{aligned}
& \vartheta_{1}(n, q)=\left[J_{n}^{\prime}\left(k_{\rho_{d}} b\right) H_{n}^{(2)^{\prime}}\left(k_{\rho_{d}} a\right)-J_{n}^{\prime}\left(k_{\rho_{d}} a\right) H_{n}^{(2)^{\prime}}\left(k_{\rho_{d}} b\right)\right] \\
& \vartheta_{5}(n, q)=\left[J_{n}\left(k_{\rho_{d}} b\right) H_{n}^{(2)}\left(k_{\rho_{d}} a\right)-J_{n}\left(k_{\rho_{d}} a\right) H_{n}^{(2)}\left(k_{\rho_{d}} b\right)\right]
\end{aligned}
$$

The electromagnetic fields within the cavity are obtained substituting (16), (17) into (6)-(11), and taking the appropriate inverse transforms. 


\subsection{External region}

The external region consists of an equivalent surface magnetic current $\left(-\bar{M}_{S}\right)$ over an infinite and perfect electric conductor cylinder of radius $b$. The external electromagnetic fields can also be written as a sum of $T E_{z}$ and $T M_{z}$ components (Harrington,1961), with vector potentials expanded in cylindrical waves, satisfying the radiation condition:

$$
\begin{aligned}
& A_{z o}(\rho, \phi, z)=\sum_{n=-\infty}^{\infty} \int_{-\infty}^{\infty} \widetilde{A}_{z o}^{e f}\left(\rho, n, k_{z}\right) e^{-j k_{z} z} e^{-j n \phi} d k_{z} \\
& F_{z o}(\rho, \phi, z)=\sum_{n=-\infty}^{\infty} \int_{-\infty}^{\infty} \widetilde{F}_{z o}^{e f}\left(\rho, n, k_{z}\right) e^{-j k_{z} z} e^{-j n \phi} d k_{z}
\end{aligned}
$$

where the superscripts ef refer to an exponential series in $\phi$ and a Fourier transform in $z$. And the vector potentials in spectral domain are given by:

$$
\begin{aligned}
& \widetilde{A}_{z o}^{e f}\left(\rho, n, k_{z}\right)=\frac{1}{(2 \pi)^{2}} \int_{-\infty}^{\infty} \int_{-\pi}^{\pi} A_{z o}(\rho, \phi, z) e^{j k_{z} z} e^{j n \phi} d \phi d k_{z} \\
& \widetilde{F}_{z o}^{e f}\left(\rho, n, k_{z}\right)=\frac{1}{(2 \pi)^{2}} \int_{-\infty}^{\infty} \int_{-\pi}^{\pi} F_{z o}(\rho, \phi, z) e^{j k_{z} z} e^{j n \phi} d \phi d k_{z}
\end{aligned}
$$

In space domain the fields are given by (5) with $\varepsilon_{d}, \mu_{d}$ and $k_{d}$ substituted by $\varepsilon_{0}, \mu_{0}$ and $k_{o}=$ $\omega \sqrt{\varepsilon_{0} \mu_{0}}$, respectively. In the transform domain the components of the electromagnetic fields are given by:

$$
\begin{aligned}
\widetilde{E}_{\rho}^{e f}\left(\rho, n, k_{z}\right) & =-\frac{k_{z}}{\omega \varepsilon_{o}} \frac{\partial \widetilde{A}_{z o}^{e f}}{\partial \rho}+\frac{j n}{\rho} \widetilde{F}_{z o}^{e f} \\
\widetilde{E}_{\phi}^{e f}\left(\rho, n, k_{z}\right) & =-\frac{n k_{z}}{j \omega \varepsilon_{o} \rho} \widetilde{A}_{z o}^{e f}+\frac{\partial \widetilde{F}_{z o}^{e f}}{\partial \rho} \\
\widetilde{E}_{z}^{e f}\left(\rho, n, k_{z}\right) & =\frac{k_{\rho_{o}}^{2}}{j \omega \varepsilon_{o}} \widetilde{A}_{z o}^{e f} \\
\widetilde{H}_{\rho}^{e f}\left(\rho, n, k_{z}\right) & =-\frac{j n}{\rho} \widetilde{A}_{z o}^{e f}-\frac{k_{z}}{\omega \mu_{o}} \frac{\partial \widetilde{F}_{z o}^{e f}}{\partial \rho} \\
\widetilde{H}_{\phi}^{e f}\left(\rho, n, k_{z}\right) & =-\frac{\partial \widetilde{A}_{z o}^{e f}}{\partial \rho}-\frac{n k_{z}}{j \omega \mu_{o} \rho} \widetilde{F}_{z o}^{e f} \\
\widetilde{H}_{z}^{e f}\left(\rho, n, k_{z}\right) & =\frac{k_{\rho_{o}}^{2}}{j \omega \mu_{o}} \widetilde{F}_{z o}^{e f}
\end{aligned}
$$

where $k_{\rho_{o}}^{2}=\left(k_{o}^{2}-k_{z}^{2}\right)$. In spectral domain:

$$
\begin{aligned}
& \widetilde{A}_{z o}^{e f}=c_{3} H_{n}^{(2)}\left(k_{\rho_{o}} \rho\right) \\
& \widetilde{F}_{z o}^{e f}=c_{4} H_{n}^{(2)}\left(k_{\rho_{o}} \rho\right)
\end{aligned}
$$

The boundary conditions at $\rho=b$ require that the equivalent surface magnetic current $-\bar{M}_{S}(\phi, z)=-\hat{n} \times \bar{E}(b, \phi, z)=-\hat{a}_{\rho} \times \bar{E}(b, \phi, z)$, where $\hat{n}$ is the unit vector normal to the 
surface $\rho=b$ pointing outwards. Consequently $M_{z}(\phi, z)=E_{\phi}(b, \phi, z)$ and $M_{\phi}(\phi, z)=$ $-E_{z}(b, \phi, z)$. In spectral domain it reads:

$$
\begin{aligned}
& \widetilde{M}_{z}^{e f}(n, q)=\widetilde{E}_{\phi}^{e f}\left(\rho, n, k_{z}\right)=-\frac{n k_{z}}{j \omega \varepsilon_{o} b} \widetilde{A}_{z o}^{e f}+\frac{\partial \widetilde{F}_{z o}^{e f}}{\partial \rho} \\
& \widetilde{M}_{\phi}^{e f}(n, q)=-\widetilde{E}_{z}^{e f}\left(\rho, n, k_{z}\right)=-\frac{k_{\rho o}^{2}}{j \omega \varepsilon_{o}} \widetilde{A}_{z o}^{e f}
\end{aligned}
$$

For a given magnetic surface current the coefficients $c_{3}$ and $c_{4}$ are determined substituting (33) and (34) into (35) and (36), and the potentials are given by:

$$
\begin{aligned}
& \widetilde{A}_{z o}^{e f}\left(\rho, n, k_{z}\right)=\widetilde{G}_{M \phi}^{A o}\left(\rho, n, k_{z}\right) \widetilde{M}_{\phi}^{e f}\left(n, k_{z}\right) \\
& \widetilde{F}_{z o}^{e f}\left(\rho, n, k_{z}\right)=\widetilde{G}_{M \phi}^{F o}\left(\rho, n, k_{z}\right) \widetilde{M}_{\phi}^{e f}\left(n, k_{z}\right)+\widetilde{G}_{M z}^{F o}\left(\rho, n, k_{z}\right) \widetilde{M}_{z}^{e f}\left(n, k_{z}\right)
\end{aligned}
$$

where:

$$
\begin{aligned}
& \widetilde{G}_{M \phi}^{A o}\left(\rho, n, k_{z}\right)=-\frac{j \omega \varepsilon_{o}}{k_{\rho_{o}}^{2}} \frac{H_{n}^{(2)}\left(k_{\rho_{o}} \rho\right)}{H_{n}^{(2)}\left(k_{\rho_{o}} b\right)} \\
& \widetilde{G}_{M \phi}^{F o}\left(\rho, n, k_{z}\right)=-\frac{n k_{z}}{k_{\rho_{o}}^{3} b} \frac{H_{n}^{(2)}\left(k_{\rho_{o}} \rho\right)}{H_{n}^{(2)^{\prime}}\left(k_{\rho_{o}} b\right)} \\
& \widetilde{G}_{M z}^{F o}\left(\rho, n, k_{z}\right)=\frac{1}{k_{\rho_{o}}} \frac{H_{n}^{(2)}\left(k_{\rho_{o}} \rho\right)}{H_{n}^{(2)^{\prime}}\left(k_{\rho_{o}} b\right)}
\end{aligned}
$$

The electromagnetic fields in the external region are obtained substituting (37)-(38) into (27)-(32), and taking the inverse transform.

\section{Feeding network}

The wraparound antenna is fed by a parallel network of equally spaced $N_{p}$ coaxial cables along a circumference $\left(z=z_{f}, \rho=b\right)$ of the antenna. An example of the feeding network, with 6 coaxial cables is shown in Fig. 2. Each probe is modeled as a strip at $z=z_{f}$, from $\rho=a$ to $\rho=b$, centered at $\phi_{f}(i)=\left[\phi_{f o}+(i-1) 2 \pi / N_{p}\right], i=1, \ldots, N_{p}$, and with angular width $\Delta \phi_{f}$. The strip $i$ is limited from $\phi_{1 f}(i)$ to $\phi_{2 f}(i)$ :

$$
\begin{aligned}
& \phi_{1 f}(i)=\phi_{f}(i)-\frac{\Delta \phi_{f}}{2} \\
& \phi_{2 f}(i)=\phi_{f}(i)+\frac{\Delta \phi_{f}}{2}
\end{aligned}
$$

It should be observed that the widths of the strips $\left(=\rho \Delta \phi_{f}\right)$ vary with the radius $\rho$, but for practical cases of thin dielectrics they become almost constant. A mean width of the strip values $W_{f}=(a+b) \Delta \phi_{f} / 2$. The total feeding volume current distribution, in $\rho$-direction, is given by:

$$
J_{f}(\rho, \phi, z)=\sum_{i=1}^{N_{p}} J_{f i}(\rho, \phi, z)
$$




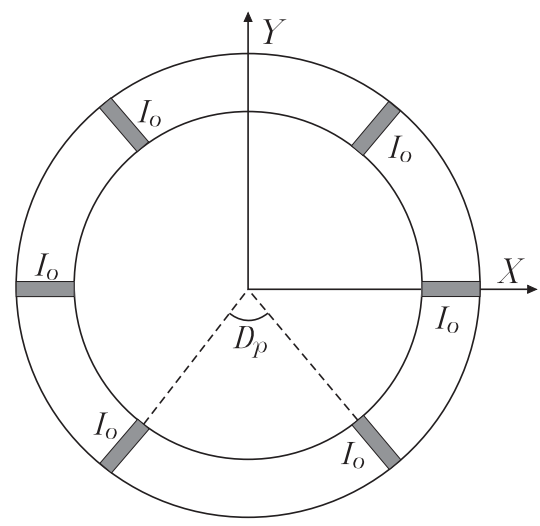

Fig. 2. Example of feeding network with 6 probes.

where

$$
J_{f i}(\rho, \phi, z)=\frac{I_{o}}{\rho \Delta \phi_{f}} \delta\left(z-z_{f}\right) \quad \phi_{1 f}(i)<\phi<\phi_{2 f}(i) \quad a<\rho<b
$$

and $I_{0}$ is the amplitude of the current on each probe. For each probe the coefficients of the exponential series in $\phi$ and sine series in $z$ are equal to:

$$
\widetilde{J}_{f i}^{e s}(\rho, n, q)=\frac{I_{o}}{\pi L_{z c} \rho} \sin \left[\frac{q \pi}{L_{z c}}\left(z_{f}-z_{1 c}\right)\right] \operatorname{sinc}\left(\frac{n \Delta \phi_{f}}{2 \pi}\right) e^{j n \phi_{f}(i)}
$$

where $\operatorname{sinc}(x)=\sin (\pi x) /(\pi x)$.

As each feed point carries a current $I_{0}$, the total current in the network is $N_{p} I_{0}$.

\section{Basis-functions}

Following the method of moments procedure the equivalent surface magnetic currents are expanded in a set of basis-functions. These currents extend from $z_{1 c}$ to $z_{1 a}$, and from $z_{2 a}$ to $z_{2 c}$, over the electric conductor introduced by the equivalence principle. Each component of the magnetic current is expanded in exponential Fourier series in $\phi$-direction, and in sub-domain basis-functions in $z$-direction, forming a piecewise-linear approximation for $M_{z}$ and a piecewise-constant one for $M_{\phi}$.

The boundary conditions require that $\phi$-component of the electric field be zero at the cavity limits $\left(z_{1 c}\right.$ and $\left.z_{2 c}\right)$, which implies that $M_{z}=0$ at these points. The piecewise-linear basis-functions used for $M_{z}$ are shown in Fig. 3, and are composed by basis-functions at

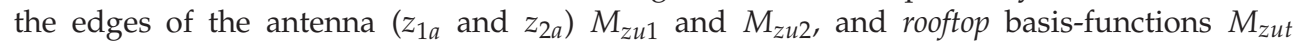
$\left(3 \leq t \leq T_{z}+2\right)$ :

$$
M_{z}(\phi, z)=\sum_{u=-U}^{U} \sum_{t=1}^{T_{z}+2} c_{u t} M_{z u t}(\phi, z)
$$

where $U$ is the maximum number of harmonics in $\phi, T_{z}$ is the number of rooftop basis-functions, and $c^{\prime} s$ are the coefficients to be determined. These basis-functions are shown in Fig. 4. The basis-functions at the edges of the antenna are given by:

$$
M_{z u 1}(\phi, z)=f_{1}(z) e^{-j u \phi}
$$




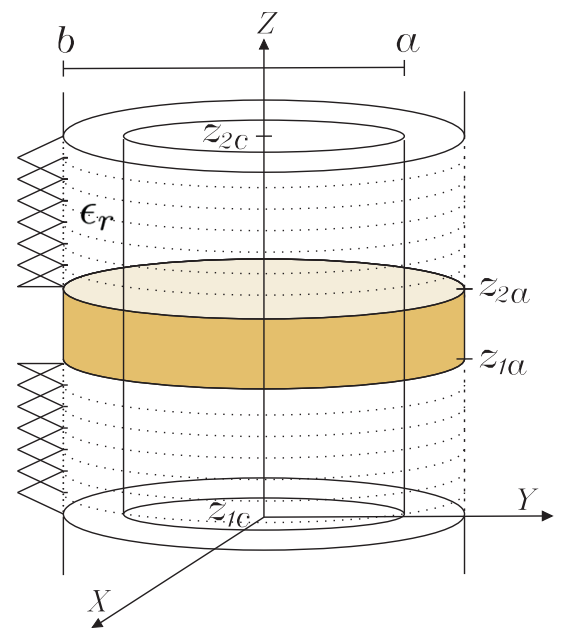

Fig. 3. Set of basis-functions for $M_{z}$.
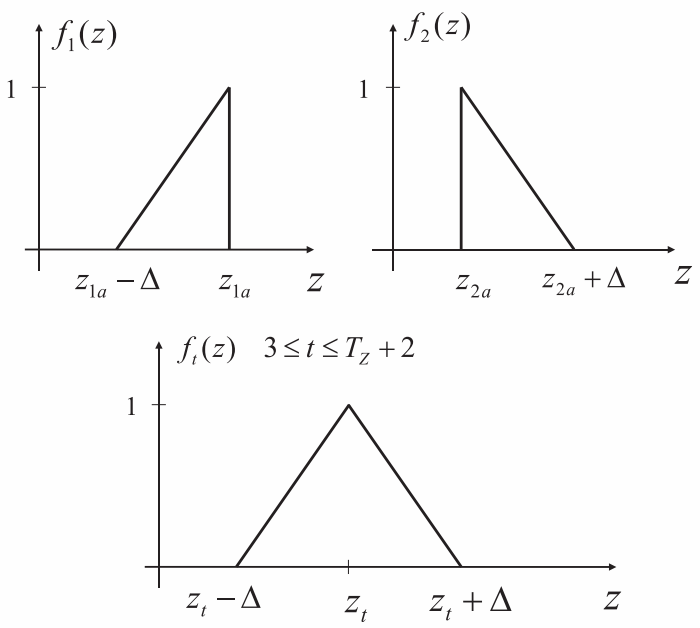

Fig. 4. Basis-functions for $M_{z}$. 


$$
M_{z u 2}(\phi, z)=f_{2}(z) e^{-j u \phi}
$$

where:

$$
\begin{aligned}
& f_{1}(z)= \begin{cases}\frac{z-z_{1 a}}{\Delta}+1 & \left(z_{1 a}-\Delta\right)<z<z_{1 a} \\
0 & \text { otherwise }\end{cases} \\
& f_{2}(z)= \begin{cases}\frac{z_{2 a}-z}{\Delta}+1 & z_{2 a}<z<\left(z_{2 a}+\Delta\right) \\
0 & \text { otherwise }\end{cases}
\end{aligned}
$$

and $\Delta$ is the segment length in $z$-direction. The rooftop functions $\left(3 \leq t \leq T_{z}+2\right)$ are given by:

$$
M_{z u t}(\phi, z)=f_{t}(z) e^{-j u \phi}
$$

where

$$
f_{t}(z)= \begin{cases}1+\frac{z-z_{t}}{\Delta} & z_{t}-\Delta<z<z_{t} \\ 1+\frac{z_{t}-z}{\Delta} & z_{t}<z<z_{t}+\Delta \\ 0 & \text { otherwise }\end{cases}
$$

and $z_{t}$ is the center coordinate of the rooftop basis-function.

Some transforms of these $z$-directed basis-functions will be needed throughout the chapter, and are given below. The exponential series in $\phi$ and Fourier transform in $z$ are given by:

$$
\begin{aligned}
& \tilde{M}_{z u 1}^{e f}\left(n, k_{z}\right)=\delta_{n, u} \tilde{f}_{1}^{f}\left(k_{z}\right) \\
& \tilde{M}_{z u 2}^{e f}\left(n, k_{z}\right)=\delta_{n, u} \tilde{f}_{2}^{f}\left(k_{z}\right) \\
& \tilde{M}_{z u t}^{e f}\left(n, k_{z}\right)=\delta_{n, u} \tilde{f}_{t}^{f}\left(k_{z}\right)
\end{aligned}
$$

where $\delta_{n, u}$ is the Kronecker's delta function given by:

$$
\delta_{n, u}= \begin{cases}1 & n=u \\ 0 & n \neq u\end{cases}
$$

and

$$
\begin{aligned}
& \tilde{f}_{1}^{f}\left(k_{z}\right)=\frac{e^{j k_{z} z_{1 a}}}{2 \pi}\left(\frac{1}{j k_{z}}+\frac{1}{\Delta k_{z}^{2}}\right)-\frac{e^{j k_{z}\left(z_{1 a}-\Delta\right)}}{2 \pi \Delta k_{z}^{2}} \\
& \widetilde{f}_{2}^{f}\left(k_{z}\right)=\frac{e^{j k_{z} z_{2 a}}}{2 \pi}\left(-\frac{1}{j k_{z}}+\frac{1}{\Delta k_{z}^{2}}\right)-\frac{e^{j k_{z}\left(z_{2 a}+\Delta\right)}}{2 \pi \Delta k_{z}^{2}} \\
& \widetilde{f}_{t}^{f}\left(k_{z}\right)=\frac{e^{j k_{z} z_{t}}}{\pi \Delta k_{z}^{2}}\left[1-\cos \left(k_{z} \Delta\right)\right]
\end{aligned}
$$

Similarly the exponential series in $\phi$ and sine series in $z$ are given by:

$$
\begin{aligned}
& \tilde{M}_{z u 1}^{e s}\left(n, k_{z}\right)=\delta_{n, u} \widetilde{f}_{1}^{s}\left(k_{z}\right) \\
& \tilde{M}_{z u 2}^{e s}\left(n, k_{z}\right)=\delta_{n, u} \widetilde{f}_{2}^{s}\left(k_{z}\right) \\
& \tilde{M}_{z u t}^{e s}\left(n, k_{z}\right)=\delta_{n, u} \widetilde{f}_{t}^{s}\left(k_{z}\right)
\end{aligned}
$$


where

$$
\begin{aligned}
\widetilde{f}_{1}^{s}\left(k_{z}\right)= & \frac{2}{q \pi}\left\{-\cos \left(\frac{q \pi}{L_{z c}}\left(z_{1 a}-z_{1 c}\right)\right)+\right. \\
& \left.\frac{L_{z c}}{\Delta q \pi}\left[\sin \left(\frac{q \pi}{L_{z c}}\left(z_{1 a}-z_{1 c}\right)\right)-\sin \left(\frac{q \pi}{L_{z c}}\left(z_{1 a}-\Delta-z_{1 c}\right)\right)\right]\right\} \\
\widetilde{f}_{2}^{s}\left(k_{z}\right)= & \frac{2}{q \pi}\left\{\cos \left(\frac{q \pi}{L_{z c}}\left(z_{2 a}-z_{1 c}\right)\right)-\right. \\
& \left.\frac{L_{z c}}{\Delta q \pi}\left[\sin \left(\frac{q \pi}{L_{z c}}\left(z_{2 a}+\Delta-z_{1 c}\right)\right)-\sin \left(\frac{q \pi}{L_{z c}}\left(z_{2 a}-z_{1 c}\right)\right)\right]\right\} \\
\widetilde{f}_{t}^{s}\left(k_{z}\right)= & \frac{4}{L_{z c} \Delta\left(q \pi / L_{z c}\right)^{2}} \sin \left[\frac{q \pi}{L_{z c}}\left(z_{t}-z_{1 c}\right)\right]\left[1-\cos \left(\frac{q \pi \Delta}{L_{z c}}\right)\right]
\end{aligned}
$$

On the other hand the $\phi$-directed equivalent magnetic surface currents are expanded in piecewise-constant basis functions as:

$$
M_{\phi}(\phi, z)=\sum_{u=-U}^{U} \sum_{t=1}^{T_{\phi}} d_{u t} M_{\phi u t}(\phi, z)
$$

where $U$ is the maximum number of harmonics in $\phi, d^{\prime} s$ are the coefficients to be determined, and $T_{\phi}$ is the number of piecewise-constant basis-functions $M_{\phi u t}$ given by:

$$
M_{\phi u t}(\phi, z)=g_{t}(z) e^{-j u \phi}
$$

where:

$$
g_{t}(z)= \begin{cases}1 & z_{t-1}<z<z_{t} \\ 0 & \text { otherwise }\end{cases}
$$

The piecewise-constant basis-functions used for $M_{\phi}$ are shown in Fig. 5, and a single basis is shown in Fig. 6. Again we will introduce some transforms of $M_{\phi u t}$ that will be required later. The exponential series in $\phi$ and Fourier transform in $z$ is given by:

$$
\widetilde{M}_{\phi u t}^{e f}\left(n, k_{z}\right)=\delta_{n, u} \widetilde{g}_{t}^{f}\left(k_{z}\right)
$$

where

$$
\widetilde{g}_{t}^{f}\left(k_{z}\right)=\frac{e^{j k_{z} z_{t}}}{j 2 \pi k_{z}}\left(1-e^{j k_{z} \Delta}\right)
$$

And the exponential series in $\phi$ and cosine series in $z$ is given by:

$$
\tilde{M}_{\phi u t}^{e c}\left(n, k_{z}\right)=\delta_{n, u} \widetilde{g}_{t}^{c}\left(k_{z}\right)
$$

where

$$
\widetilde{g}_{t}^{c}\left(k_{z}\right)=\frac{\epsilon_{q}}{q \pi}\left\{\sin \left[\frac{q \pi}{L_{z c}}\left(z_{t}-z_{1 c}\right)\right]-\sin \left[\frac{q \pi}{L_{z c}}\left(z_{t}-\Delta-z_{1 c}\right)\right]\right\}
$$




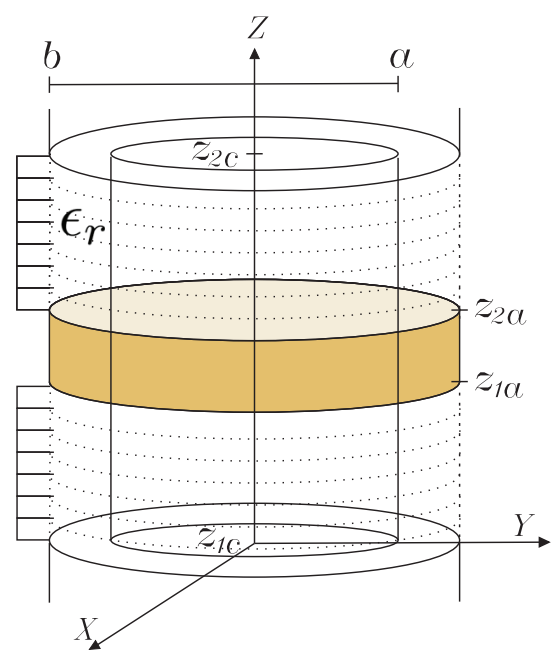

Fig. 5. Set of basis-functions for $M_{\phi}$.

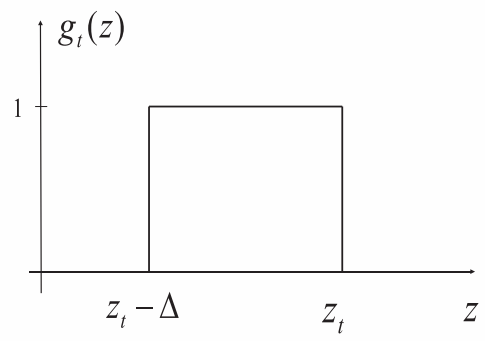

Fig. 6. Basis-function for $M_{\phi}$.

\section{Method of moments linear system}

The coefficients $c_{u t}$ and $d_{u t}$ of the expansions of magnetic surface currents (47) and (67) are determined in order to satisfy the boundary conditions. The continuity of the tangential electric field is already guaranteed as the equivalent magnetic surface currents in the inner and outer regions have opposite signs. And the continuity of the tangential components of the magnetic field over the interface between the inner and outer regions imply:

$$
\underbrace{H^{<}\left(M_{z}\right)+H^{<}\left(M_{\phi}\right)+H^{<}\left(J_{f}\right)}_{H^{<}}=\underbrace{H^{>}\left(M_{z}\right)+H^{>}\left(M_{\phi}\right)}_{H^{>}}
$$

for the $z$ and $\phi$-components, over the surface $\rho=b, z_{1 c}<z<z_{1 a}$ and $z_{2 a}<z<z_{2 c}$. The superscript $<$ refers to the cavity region, and $>$ refers to the outer region. For $H_{z}$, using the 
current expansions (47) and (67):

$$
\sum_{u=-U}^{U} \sum_{t=1}^{T_{z}+2} c_{u t}\left[H_{z}^{<}\left(M_{z u t}\right)-H_{z}^{>}\left(M_{z u t}\right)\right]+\sum_{u=-U}^{U} \sum_{t=1}^{T_{\phi}} d_{u t}\left[H_{z}^{<}\left(M_{\phi u t}\right)-H_{z}^{>}\left(M_{\phi u t}\right)\right]=-H_{z}^{<}\left(J_{f}\right)
$$

Defining the symmetric product:

$$
<f, g>=\int_{0}^{2 \pi} \int_{-\infty}^{+\infty} f(\phi, z) g(\phi, z) b d \phi d z
$$

and taking the symmetric product of (75) by testing functions which are chosen as the same set of basis-functions $M_{z m s}(\phi, z),-U \leq m \leq+U, s=1, \ldots, T_{z}+2$ results:

$$
\begin{array}{r}
\sum_{u=-U}^{U} \sum_{t=1}^{T_{z}+2} c_{u t}<H_{z}^{<}\left(M_{z u t}\right)-H_{z}^{>}\left(M_{z u t}\right), M_{z m s}>+ \\
\sum_{u=-U}^{U} \sum_{t=1}^{T_{\phi}} d_{u t}<H_{z}^{<}\left(M_{\phi u t}\right)-H_{z}^{>}\left(M_{\phi u t}\right), M_{z m s}>=-<H_{z}^{<}\left(J_{f}\right), M_{z m s}> \\
-U \leq m \leq+U, s=1, \ldots, T_{z}+2
\end{array}
$$

Similarly for $H_{\phi}$, using the current expansions (47) and (67) and taking the symmetric product of (75) by testing functions which are chosen as the same set of basis-functions $M_{\phi m s}(\phi, z),-U \leq m \leq+U, s=1, \ldots, T_{\phi}$ results:

$$
\begin{gathered}
\sum_{u=-U}^{U} \sum_{t=1}^{T_{z}+2} c_{u t}<H_{\phi}^{<}\left(M_{z u t}\right)-H_{\phi}^{>}\left(M_{z u t}\right), M_{\phi m s}>+ \\
\sum_{u=-U}^{U} \sum_{t=1}^{T_{\phi}} d_{u t}<H_{\phi}^{<}\left(M_{\phi u t}\right)-H_{\phi}^{>}\left(M_{\phi u t}\right), M_{\phi m s}>=-<H_{\phi}^{<}\left(J_{f}\right), M_{\phi m s}> \\
-U \leq m \leq+U, s=1, \ldots, T_{\phi}
\end{gathered}
$$

Re-arranging (77) and (78) in a matricial form:

$$
\left[\begin{array}{ccc}
{\left[Z^{z z}\right]} & \vdots & {\left[Z^{z \phi}\right]} \\
\cdots & \cdots & \cdots \\
{\left[Z^{\phi z}\right]} & \vdots & {\left[Z^{\phi \phi}\right]}
\end{array}\right]\left[\begin{array}{l}
{[c]} \\
\cdots \\
{[d]}
\end{array}\right]=\left[\begin{array}{c}
{\left[V^{z}\right]} \\
\cdots \\
{\left[V^{\phi}\right]}
\end{array}\right]
$$

where $[c]$ and $[d]$ are arrays which elements are the coefficients $c_{u t}$ and $d_{u t}$, respectively. And the elements of the submatrices are given by:

$$
\begin{aligned}
Z_{m s, u t}^{z z} & =<H_{z}^{<}\left(M_{z u t}\right), M_{z m s}>-<H_{z}^{>}\left(M_{z u t}\right), M_{z m s}> \\
Z_{m s, u t}^{z \phi} & =<H_{z}^{<}\left(M_{\phi u t}\right), M_{z m s}>-<H_{z}^{>}\left(M_{\phi u t}\right), M_{z m s}> \\
Z_{m s, u t}^{\phi z} & =<H_{\phi}^{<}\left(M_{z u t}\right), M_{\phi m s}>-<H_{\phi}^{>}\left(M_{z u t}\right), M_{\phi m s}> \\
Z_{m s, u t}^{\phi \phi} & =<H_{\phi}^{<}\left(M_{\phi u t}\right), M_{\phi m s}>-<H_{\phi}^{>}\left(M_{\phi u t}\right), M_{\phi m s}> \\
V_{m s}^{z} & =-<H_{z}^{<}\left(J_{f}\right), M_{z m s}> \\
V_{m s}^{\phi} & =-<H_{\phi}^{<}\left(J_{f}\right), M_{\phi m s}>
\end{aligned}
$$


Each symmetric product above represents the reaction of the field due to a basis-function, or due to the feed, on a testing function. The procedure will be illustrated for the elements of the submatrix $\left[Z^{z z}\right]$. The field $H_{z}$ due to $M_{z u t}$ in the cavity is given by the inverse transform of (11), with the vector potential given by (17):

$$
H_{z}^{<}\left(M_{z u t}\right)(\rho, \phi, z)=\sum_{n=-\infty}^{\infty} \sum_{q=1}^{\infty}\left[\frac{\left(k_{\rho_{d}}\right)^{2}}{j \omega \mu_{d}} \widetilde{G}_{M z}^{F d}(\rho, n, q) \widetilde{M}_{z u t}^{e s}(n, q)\right] e^{-j n \phi} \sin \left[\frac{q \pi}{L_{z c}}\left(z-z_{1 c}\right)\right]
$$

And the field $H_{z}$ due to $M_{z u t}$ outside the cylinder is given by the inverse transform of (32) with the vector potential given by (38):

$$
H_{z}^{>}\left(M_{z u t}\right)(\rho, \phi, z)=\sum_{n=-\infty}^{\infty} \int_{-\infty}^{\infty}\left[\frac{\left(k_{\rho_{o}}\right)^{2}}{j \omega \mu_{o}} \widetilde{G}_{M z}^{F o}\left(\rho, n, k_{z}\right) \tilde{M}_{z u t}^{e f}\left(n, k_{z}\right)\right] e^{-j k_{z} z} e^{-j n \phi} d k_{z}
$$

Substituting (86) and (87) into (80) after some manipulations we obtain:

$$
\begin{aligned}
Z_{m s, u t}^{z z}= & \delta_{-u, m} \frac{b \pi L_{z c}}{j \omega \mu_{d}} \sum_{q=1}^{\infty}\left(k_{\rho_{d}}\right)^{2} \widetilde{G}_{M z}^{F d}(b, u, q) \widetilde{f}_{t}^{s}(q) \widetilde{f}_{s}^{s}(q)- \\
& \delta_{-u, m} \frac{(2 \pi)^{2} b}{j \omega \mu_{o}} \int_{-\infty}^{\infty}\left(k_{\rho_{o}}\right)^{2} \widetilde{G}_{M z}^{F o}\left(b, u, k_{z}\right) \widetilde{f}_{t}^{f}\left(k_{z}\right) \widetilde{f}_{s}^{f}\left(-k_{z}\right) d k_{z}
\end{aligned}
$$

Similarly for the elements of submatrices $Z^{z \phi}, Z^{\phi z}$ and $Z^{\phi \phi}$ :

$$
\begin{gathered}
Z_{m s, u t}^{z \phi}=\delta_{-u, m} \frac{b \pi L_{z c}}{j \omega \mu_{d}} \sum_{q=1}^{\infty}\left(k_{\rho_{d}}\right)^{2} \widetilde{G}_{M \phi}^{F d}(b, u, q) \widetilde{g}_{t}^{c}(q) \widetilde{f}_{s}^{s}(q)- \\
\delta_{-u, m} \frac{(2 \pi)^{2} b}{j \omega \mu_{o}} \int_{-\infty}^{\infty}\left(k_{\rho_{o}}\right)^{2} \widetilde{G}_{M \phi}^{F o}\left(b, u, k_{z}\right) \widetilde{g}_{t}^{f}\left(k_{z}\right) \widetilde{f}_{s}^{f}\left(-k_{z}\right) d k_{z} \\
Z_{m s, u t}^{\phi z}=-\delta_{-u, m} \frac{u \pi^{2}}{\omega \mu_{d}} \sum_{q=1}^{\infty} q \widetilde{G}_{M z}^{F d}(b, u, q) \widetilde{f}_{t}^{s}(q) \widetilde{g}_{s}^{c}(q)+ \\
\delta_{-u, m} \frac{(2 \pi)^{2} u}{j \omega \mu_{o}} \int_{-\infty}^{\infty} k_{z} \widetilde{G}_{M z}^{F o}\left(b, u, k_{z}\right) \widetilde{f}_{t}^{f}\left(k_{z}\right) \widetilde{g}_{s}^{f}\left(-k_{z}\right) d k_{z} \\
\delta_{-u, m} 2 \pi b L_{z c} \sum_{q=0}^{\infty} \frac{1}{\epsilon_{q}}\left[-\frac{\partial}{\partial \rho} \widetilde{G}_{M \phi}^{A d}(b, u, q)-\frac{u}{\omega \mu_{d} b} \frac{q \pi}{L_{z c}} \widetilde{G}_{M \phi}^{F d}(b, u, q)\right] \widetilde{g}_{t}^{c}(q) \widetilde{g}_{s}^{c}(q)- \\
\delta_{-u, m}(2 \pi)^{2} b \int_{-\infty}^{\infty}\left[-\frac{\partial}{\partial \rho} \widetilde{G}_{M \phi}^{A o}\left(b, u, k_{z}\right)-\frac{u k_{z}}{j \omega \mu_{o} b} \widetilde{G}_{M \phi}^{F o}\left(b, u, k_{z}\right)\right] \widetilde{g}_{t}^{f}\left(k_{z}\right) \widetilde{g}_{s}^{f}\left(-k_{z}\right) d k_{z}
\end{gathered}
$$

In fact the elements of submatrix $Z^{z \phi}(81)$ are related to those of $Z^{\phi z}$ (82) by the reciprocity theorem (Harrington,1961):

$$
\begin{aligned}
Z_{m s, u t}^{z \phi} & =<H_{z}^{<}\left(M_{\phi u t}\right), M_{z m s}>-<H_{z}^{>}\left(M_{\phi u t}\right), M_{z m s}> \\
& =<M_{\phi u t}, H_{\phi}^{<}\left(M_{z m s}\right)>-<M_{\phi u t}, H_{\phi}^{>}\left(M_{z m s}\right)> \\
& =<H_{\phi}^{<}\left(M_{z m s}\right), M_{\phi u t}>-<H_{\phi}^{>}\left(M_{z m s}\right), M_{\phi u t}> \\
& =Z_{u t, m s}^{\phi z}
\end{aligned}
$$


Reciprocity theorem can also be used to calculate the elements of the voltage vector $[V]$. For example the elements of subvector $\left[V^{z}\right]$ given by (84) can be written as:

$$
\begin{aligned}
V_{m s}^{z} & =-<H_{z}^{<}\left(J_{f}\right), M_{z m s}> \\
& =-\int_{0}^{2 \pi} \int_{-\infty}^{\infty} H_{z}^{<}\left(J_{f}\right)(b, \phi, z) M_{z m s}(\phi, z) b d \phi d z \\
& =\iiint_{V} E_{\rho}^{<}\left(M_{z m s}\right)(\rho, \phi, z) J_{f}(b, \phi, z) d v
\end{aligned}
$$

where the volume $V$ refers to the cavity. $E_{\rho}^{<}\left(M_{z m s}\right)(\rho, \phi, z)$ is given by the inverse transform of (6), using (16) and (17):

$$
E_{\rho}^{<}\left(M_{z m s}\right)(\rho, \phi, z)=\sum_{n=-\infty}^{\infty} \sum_{q=1}^{\infty} \frac{j n}{\rho} \widetilde{G}_{M z}^{F d}(\rho, n, q) \tilde{M}_{z m s}^{e s}(n, q) e^{-j n \phi} \sin \left[\frac{q \pi}{L_{z c}}\left(z-z_{1 c}\right)\right]
$$

Substituting (94) and (44) into (93) after some manipulations results:

$$
V_{m s}^{z}=j m \pi L_{z \mathcal{c}} \int_{a}^{b} \sum_{q=1}^{\infty} \widetilde{G}_{M z}^{F d}(\rho, m, q) \widetilde{f}_{\mathcal{s}}^{\mathcal{S}}(q) \widetilde{J}_{f}^{e s}(\rho,-m, q) d \rho
$$

Proceeding similarly for $V_{m s}^{\phi}(85)$ :

$$
\begin{aligned}
V_{m s}^{\phi} & =\iiint_{V} E_{\rho}^{<}\left(M_{\phi m s}\right)(\rho, \phi, z) J_{f}(b, \phi, z) d v \\
& =\pi L_{z c} \int_{a}^{b} \sum_{q=1}^{\infty}\left[\frac{-1}{j \omega \varepsilon_{d}} \frac{q \pi}{L_{z c}} \frac{\partial}{\partial \rho} \widetilde{G}_{M \phi}^{A d}(\rho, m, q)+\frac{j m}{b} \widetilde{G}_{M \phi}^{F d}(\rho, m, q)\right] \widetilde{g}_{s}^{c}(q) \widetilde{J}_{f}^{e s}(\rho,-m, q) \rho d \rho
\end{aligned}
$$

\section{Numerical considerations}

The method of moments linear system (79) requires the evaluation of Bessel functions of a wide range of arguments and orders, which is limited by the accuracy of the numerical routines, or by the intrinsic behavior of these functions. This limitation is enhanced for larger radius (Erturk \& Rojas,2002; Erturk \& Rojas,2003), as the arguments of the Bessel functions are proportional to either $a$ or $b$. In order to mitigate this problem the Green's functions have been written in such a way that the Bessel functions appear in specific combinations as (21), (22) and:

$$
\begin{aligned}
& \vartheta_{3}(n, q)=\left[J_{n}\left(k_{\rho_{d}} b\right) H_{n}^{(2)^{\prime}}\left(k_{\rho_{d}} a\right)-J_{n}^{\prime}\left(k_{\rho_{d}} a\right) H_{n}^{(2)}\left(k_{\rho_{d}} b\right)\right] \\
& \vartheta_{7}(n, q)=\left[J_{n}^{\prime}\left(k_{\rho_{d}} b\right) H_{n}^{(2)}\left(k_{\rho_{d}} a\right)-J_{n}\left(k_{\rho_{d}} a\right) H_{n}^{(2)^{\prime}}\left(k_{\rho_{d}} b\right)\right] \\
& \vartheta_{9}(n, q)=\frac{H_{n}^{(2)}\left(k_{\rho_{d}} b\right)}{H_{n}^{(2)^{\prime}}\left(k_{\rho_{d}} b\right)}
\end{aligned}
$$

These combinations are evaluated differently according to the argument range:

(i) For very small arguments analytical expressions of such combinations are obtained from 
the limits of the Bessel functions (Harrington,1961):

$$
\begin{aligned}
& \lim _{|z| \rightarrow 0} J_{n}(z)= \begin{cases}1-\frac{z^{2}}{4} & n=0 \\
\frac{1}{n !}\left(\frac{z}{2}\right)^{n} & n>0\end{cases} \\
& \lim _{|z| \rightarrow 0} Y_{n}(z)= \begin{cases}\frac{2}{\pi} \ln \left(\frac{\gamma z}{2}\right) & n=0 \\
-\frac{(n-1) !}{\pi}\left(\frac{2}{z}\right)^{n} & n>0\end{cases}
\end{aligned}
$$

where $\gamma=1.781072418 \ldots$. Substituting into (21), (22), (98)-(100):

$$
\begin{aligned}
& \lim _{\left|k_{\rho_{d}} a\right| \rightarrow 0} \vartheta_{1}(n, q)= \begin{cases}\frac{j}{\pi}\left(\frac{b}{a}-\frac{a}{b}\right) & n=0 \\
\frac{j n}{\pi k_{\rho_{d}}^{2}}\left[-\frac{1}{a^{2}}\left(\frac{b}{a}\right)^{n-1}+\frac{1}{b^{2}}\left(\frac{a}{b}\right)^{n-1}\right] & n>0\end{cases} \\
& \lim _{\left|k_{\rho_{d}} a\right| \rightarrow 0} \vartheta_{3}(n, q)= \begin{cases}-\frac{2 j}{\pi}\left[\frac{1}{k_{\rho_{d}} a}+\frac{k_{\rho_{d}} a}{2} \ln \left(\frac{\gamma}{2} k_{\rho_{d}} b\right)\right] & n=0 \\
-\frac{j}{\pi k_{\rho_{d}}}\left[\frac{1}{a}\left(\frac{b}{a}\right)^{n}+\frac{1}{b}\left(\frac{a}{b}\right)^{n-1}\right] & n>0\end{cases} \\
& \lim _{\left|k_{\rho_{d}} a\right| \rightarrow 0} \vartheta_{5}(n, q)= \begin{cases}\frac{2 j}{\pi} \ln \left(\frac{b}{a}\right) & n=0 \\
\frac{j}{n \pi}\left[\left(\frac{b}{a}\right)^{n}-\left(\frac{a}{b}\right)^{n}\right] & n>0\end{cases} \\
& \lim _{\left|k_{\rho_{d}} a\right| \rightarrow 0} \vartheta_{7}(n, q)= \begin{cases}\frac{2 j}{\pi}\left[\frac{1}{k_{\rho_{d}} b}+\frac{k_{\rho_{d}} b}{2} \ln \left(\frac{\gamma}{2} k_{\rho_{d}} a\right)\right] & n=0 \\
\frac{j}{\pi k_{\rho_{d}}}\left[\frac{1}{a}\left(\frac{b}{a}\right)^{n-1}+\frac{1}{b}\left(\frac{a}{b}\right)^{n}\right] & n>0\end{cases} \\
& \lim _{\left|k_{\rho_{d}} b\right| \rightarrow 0} \vartheta_{9}(n, q)= \begin{cases}\frac{j \pi}{2} k_{\rho_{d}} b+k_{\rho_{d}} b \ln \left(\frac{\gamma}{2} k_{\rho_{d}} b\right) & n=0 \\
-\frac{k_{\rho_{d}} b}{n} & n>0\end{cases}
\end{aligned}
$$

These analytical limits can be evaluated for arguments much smaller than the Bessel functions individually, due to opposite behavior of the Bessel functions in these combinations. The maximum magnitude of the argument $\left|z_{1}\right|$ for which the limits above should be used depends on $n$. A simple expression for this threshold can be found to ensure that the magnitude of the Bessel functions are larger than $10^{-\operatorname{Exp} 1}$ and smaller than $10^{+\operatorname{Exp} 1}$, where Exp 1 depends on the precision used:

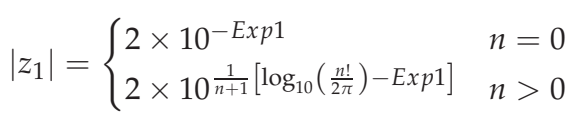

(ii) For very large arguments asymptotic expressions of Bessel functions (Harrington,1961) are used to obtain analytical expressions of the combinations.

$$
\begin{aligned}
& \lim _{|z| \rightarrow \infty} J_{n}(z)=\sqrt{\frac{2}{\pi z}} \cos (z-n \pi / 2-\pi / 4) \\
& \lim _{|z| \rightarrow \infty} Y_{n}(z)=\sqrt{\frac{2}{\pi z}} \sin (z-n \pi / 2-\pi / 4)
\end{aligned}
$$


Substituting into (21), (22), (98)-(100):

$$
\begin{aligned}
\lim _{\left|k_{\rho_{d}} b\right| \rightarrow \infty} \vartheta_{1}(n, q) & =\frac{2 j}{\pi k_{\rho_{d}} \sqrt{a b}}\left\{\left(1+\frac{1}{4 k_{\rho_{d}}^{2} a b}\right) \sin \left[k_{\rho_{d}}(b-a)\right]+\frac{1}{2 k_{\rho_{d}}}\left(\frac{1}{b}-\frac{1}{a}\right) \cos \left[k_{\rho_{d}}(b-a)\right]\right\} \\
\lim _{\left|k_{\rho_{d}} b\right| \rightarrow \infty} \vartheta_{3}(n, q) & =-\frac{2 j}{\pi k_{\rho_{d}} \sqrt{a b}}\left\{\frac{1}{2 k_{\rho_{d}} a} \sin \left[k_{\rho_{d}}(b-a)\right]+\cos \left[k_{\rho_{d}}(b-a)\right]\right\} \\
\lim _{\left|k_{\rho_{d}} b\right| \rightarrow \infty} \vartheta_{5}(n, q) & =\frac{2 j}{\pi k_{\rho_{d}} \sqrt{a b}} \sin \left[k_{\rho_{d}}(b-a)\right] \\
\lim _{\left|k_{\rho_{d}} b\right| \rightarrow \infty} \vartheta_{7}(n, q) & =\frac{2 j}{\pi k_{\rho_{d}} \sqrt{a b}}\left\{\cos \left[k_{\rho_{d}}(b-a)\right]-\frac{1}{2 k_{\rho_{d}} b} \sin \left[k_{\rho_{d}}(b-a)\right]\right\} \\
\lim _{\left|k_{\rho_{d}} b\right| \rightarrow \infty} \vartheta_{9}(n, q) & =j
\end{aligned}
$$

The use of these asymptotic limits are specially important for the case when the imaginary part of the arguments are large and the Bessel functions present an exponential behavior. In this case asymptotic limits of the combinations also present an exponentially behavior, but the exponent is no longer proportional to the cylinder radius, but to the dielectric thickness, allowing the combinations to be calculated for much larger arguments. The minimum value of the magnitude of the argument $\left(\left|z_{2}\right|\right)$ from which the asymptotic limits are used is specified depending on the precision used.

(iii) Numerical routines of Bessel functions are only used if the magnitude of the arguments lies between $\left|z_{1}\right|$ and $\left|z_{2}\right|$, where they present good convergence.

This procedure avoids that the numerical routines result in overflow or underflow.

\section{Input impedance}

The input impedance observed in a single coaxial cable, for example cable 1 , is given by the variational expression (Harrington,1961):

$$
Z_{\text {in }}=-\frac{1}{I_{0}^{2}} \iiint_{V}\left[\vec{E}(\vec{M})+\vec{E}\left(\vec{J}_{f}\right)\right] \cdot \vec{J}_{f 1} d v
$$

where $\bar{M}$ represents the equivalent magnetic surface current distribution, including $z$ and $\phi$ components, and $\bar{J}_{f}$ is the total network feed current distribution. Isolating initially the component due to $\bar{M}$, and using the basis expansions (47) and (67):

$$
\begin{gathered}
Z_{i n}^{M}=-\frac{1}{I_{O}^{2}} \sum_{u=-U}^{U} \sum_{t=1}^{T_{z}+2} c_{u t} \iiint_{V} E_{\rho}\left(M_{z u t}\right)(\rho, \phi, z) J_{f 1}(\rho, \phi, z) d v- \\
\frac{1}{I_{O}^{2}} \sum_{u=-U}^{U} \sum_{t=1}^{T_{\phi}} d_{u t} \iiint_{V} E_{\rho}\left(M_{\phi u t}\right)(\rho, \phi, z) J_{f 1}(\rho, \phi, z) d v
\end{gathered}
$$

As the coaxial cables are equally spaced and fed with the same current $I_{0}$, the same input impedance is observed at any of the coaxial cables, and we can write: 


$$
\begin{aligned}
N_{p} Z_{i n}^{M}=- & \frac{1}{I_{0}^{2}} \sum_{u=-U}^{U} \sum_{t=1}^{T_{z}+2} c_{u t} \iiint_{V} E_{\rho}\left(M_{z u t}\right)(\rho, \phi, z) J_{f}(\rho, \phi, z) d v- \\
& \frac{1}{I_{o}^{2}} \sum_{u=-U}^{U} \sum_{t=1}^{T_{\phi}} d_{u t} \iiint_{V} E_{\rho}\left(M_{\phi u t}\right)(\rho, \phi, z) J_{f}(\rho, \phi, z) d v
\end{aligned}
$$

where $J_{f}(44)$ includes all $N_{p}$ coaxial cables. From (93) and (96) the integrals above can be identified as elements of the voltage vector of the method of moments linear system:

$$
Z_{i n}^{M}=\frac{-1}{N_{p} I_{o}^{2}} \sum_{u=-U}^{U}\left[\sum_{t=1}^{T_{z}+2} c_{u t} V_{u t}^{z}+\sum_{t=1}^{T_{\phi}} d_{u t} V_{u t}^{\phi}\right]
$$

The second portion of $Z_{\text {in }}$ (116) includes the reaction of the fields due to the feeding network on a single coaxial cable and will be called $Z_{i n}^{f}$. An approximation of this impedance will be obtained by a similar procedure to those presented at (Zheng \& Chang,1991) and (Tulintseff et. al.,1991) for self-impedance of the probe in planar dielectric layers. This approximation disregards the radiation contribution of the feed network, as if the feed is enclosed in the cavity limited by $\rho=b$, and assumes that the dielectric is thin and the fields have no variation with $\rho$. Within this cavity the $\rho$-component of the electric field is given by:

$$
\frac{1}{d^{2}} \frac{\partial^{2}}{\partial \phi^{2}} E_{\rho}+\frac{\partial^{2}}{\partial z^{2}} E_{\rho}+k_{d}^{2} E_{\rho}=j \omega \mu_{d} J_{f}
$$

where $d=(a+b) / 2$. The eigenmodes of the cavity are given by:

$$
E_{\rho m n}(\phi, z)=E_{m n} e^{j m \phi} \sin \left[\frac{n \pi}{L_{z c}}\left(z-z_{1 c}\right)\right]
$$

which is the solution of:

$$
\frac{1}{d^{2}} \frac{\partial^{2}}{\partial \phi^{2}} E_{\rho m n}+\frac{\partial^{2}}{\partial z^{2}} E_{\rho m n}+k_{m n}^{2} E_{\rho m n}=0
$$

where $k_{m n}^{2}=(m / d)^{2}+\left(n \pi / L_{z c}\right)^{2}$. And the electric field $E_{\rho}$ (120) can be expanded into eigenmodes of the cavity given by:

$$
E_{\rho}(\phi, z)=\sum_{m=-\infty}^{\infty} \sum_{n=1}^{\infty} E_{m n} e^{j m \phi} \sin \left[\frac{n \pi}{L_{z c}}\left(z-z_{1 c}\right)\right]
$$

The coefficients of the modal expansion $E_{m n}$ are determined substituting (123) into (120) and using the network feeding current (44):

$$
E_{m n}=\frac{j \omega \mu_{d} I_{o}}{\pi d L_{z c}\left[k_{d}^{2}-k_{m n}^{2}\right]} \sin \left[\frac{n \pi}{L_{z c}}\left(z_{f}-z_{1 c}\right)\right] \operatorname{sinc}\left[\frac{m \Delta_{\phi_{f}}}{2 \pi}\right] e^{-j m \phi_{f o}}\left(\sum_{i=1}^{N_{p}} e^{-j m(i-1) 2 \pi / N_{p}}\right)
$$

The approximation for $Z_{i n}^{f}$ is obtained substituting the electric field (123) and the feed (45) into:

$$
Z_{i n}^{f}=-\frac{1}{I_{o}^{2}} \iiint_{V} E_{\rho}\left(\vec{J}_{f}\right) J_{f 1} d v
$$


which results:

$$
Z_{i n}^{f}=-\frac{j \omega \mu_{d} h}{\pi d L_{z c}} \sum_{m=-\infty}^{\infty} \sum_{n=1}^{\infty} \frac{1}{k_{d}^{2}-k_{m n}^{2}} \sin ^{2}\left[\frac{n \pi}{L_{z c}}\left(z_{f}-z_{1 c}\right)\right] \operatorname{sinc}^{2}\left[\frac{m \Delta_{\phi_{f}}}{2 \pi}\right]\left(\sum_{i=1}^{N_{p}} e^{-j m(i-1) 2 \pi / N_{p}}\right)
$$

From (116), (119) and (126) the input impedance at each probe will be equal to:

$$
Z_{\text {in }}=Z_{\text {in }}^{M}+Z_{\text {in }}^{f}
$$

\section{Results}

Initially the field distribution is presented as a function of the number of feeds. The cylinder has radius of $26 \mathrm{~mm}$, and a cavity of thickness equal to $1 \mathrm{~mm}$ is filled with a dielectric of relative permitivitty of 2.1. A wraparound antenna of $34.5 \mathrm{~mm}$ (from $z_{1 a}=-17.25 \mathrm{~mm}$ to $z_{2 a}=17.25 \mathrm{~mm}$ ) is printed onto the dielectric surface. Figure 7 shows the magnitude of the electric field at $x y$ plane, at $f=3 \mathrm{GHz}$ when the antenna is fed by $1,2,3$ or 4 equally spaced probes at $z_{f}=8.625 \mathrm{~mm}$, modeled as strips of $2 \mathrm{~mm}$ of width. In this case the circumference of the antenna equals 2.4 times the dielectric wavelength. It can be observed from Fig. 7 that the pattern becomes close to omnidirectional when at least 3 probes are used. In general the spacing between the feed points of a cavity-backed wraparound antenna should be smaller than one wavelength in the dielectric substrate in order to obtain an omnidirectional pattern. But the exact number of probes will depend on the accuracy required. This is similar to that observed by (Munson,1974) for a microstrip wraparound antenna. Figure 8 shows a comparison of the magnitude of the electric field at $x z$ plane for the cavity-backed wraparound antenna obtained with this formulation, and that of a microstrip wraparound antenna obtained with cavity method. Four probes were used, which is enough to radiate an omnidirectional pattern. Despite the differences in the geometry and methods of analysis, the radiation patterns show similar behavior.

Figure 9 shows the input impedance of a cavity-backed wraparound antenna of length $L_{z c}=$ $20 \mathrm{~mm}$. The perfect cylindrical conductor has radius $b=21 \mathrm{~mm}$, and the thickness of the cavity values $(b-a)=1 \mathrm{~mm}$. It is filled with a dielectric of relative permitivitty equal to 9.6. No dielectric losses were considered. Four probes of coaxial cables were used to reach an omnidirectional pattern. They were placed at $z_{f}=5 \mathrm{~mm}$, and modeled by a strip of width equal to $1.45 \mathrm{~mm}$. The results were plotted around the frequency $2.27 \mathrm{GHz}$, which corresponds to the first omnidirectional mode. Figure 9 also shows results obtained from Ansoft HFSS, and it is observed that the input impedances from both methods are very close. The cavity effect can be observed in Fig. 10, where input impedances of the same antenna is shown for cavities of $40 \mathrm{~mm}, 50 \mathrm{~mm}$, and $60 \mathrm{~mm}$. As can be observed the cavity size has little influence on the input impedance, unless the perfect electric walls of the cavity at $z=z_{1 c}$ and $z=z_{2 c}$ becomes too close to the antenna. The effect of the dielectric thickness for the same antenna is also shown in Fig. 11. As the thickness increases the resonance frequency diminishes, and a significant bandwidth increase is observed. We can also observe a reactance shift, including the self-contribution of the probe. These properties of the cavity-backed wraparound antenna are also observed for the microstrip wraparound antenna with infinite dielectric layer.

\section{Conclusion}

This chapter has described an accurate model for cavity-backed wraparound antenna using method of moments and equivalence principle. It addresses the numerical challenges of 
$|E| \times \phi$

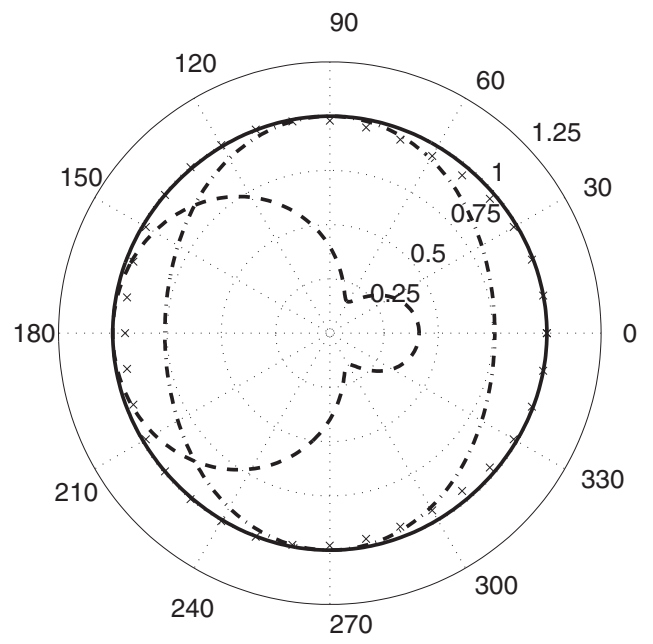

Fig. 7. $|E| \times \phi$ at $x y$ plane. - - - -: 1 feed, - . - . - .: 2 feeds, $x \times x: 3$ feeds, : 4 feeds. $f=3$ $\mathrm{GHz}, a=25 \mathrm{~mm}, h=1 \mathrm{~mm}, z_{1 c}=-25 \mathrm{~mm}, z_{2 c}=25 \mathrm{~mm}, z_{1 a}=-17.25 \mathrm{~mm}, z_{2 a}=17.25 \mathrm{~mm}, z_{f}=8.625$ $\mathrm{mm}, W_{f}=2 \mathrm{~mm}, \varepsilon_{r}=2.1$.

IEI $\times \theta$

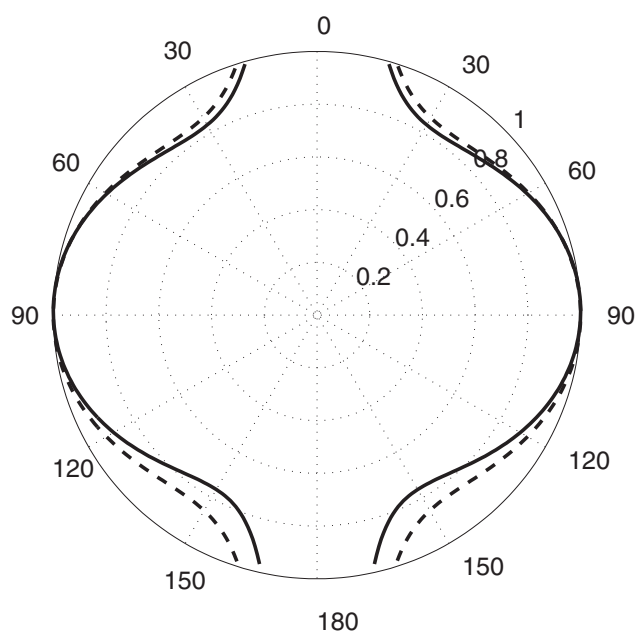

Fig. 8. $|E| \times \theta$ at $x z$ plane. : this formulation for a cavity-backed wraparound antenna, - -: cavity method for a microstrip wraparound antenna. $f=3 \mathrm{GHz}, a=25 \mathrm{~mm}, h=1 \mathrm{~mm}, z_{1 c}=-25$ $\mathrm{mm}, z_{2 c}=25 \mathrm{~mm}, z_{1 a}=-17.25 \mathrm{~mm}, z_{2 a}=17.25 \mathrm{~mm}, z_{f}=8.625 \mathrm{~mm}, W_{f}=2 \mathrm{~mm}, \varepsilon_{r}=2.1,4$ probes at $\phi=0^{\circ}, 90^{\circ}, 180^{\circ}, 270^{\circ}$. 


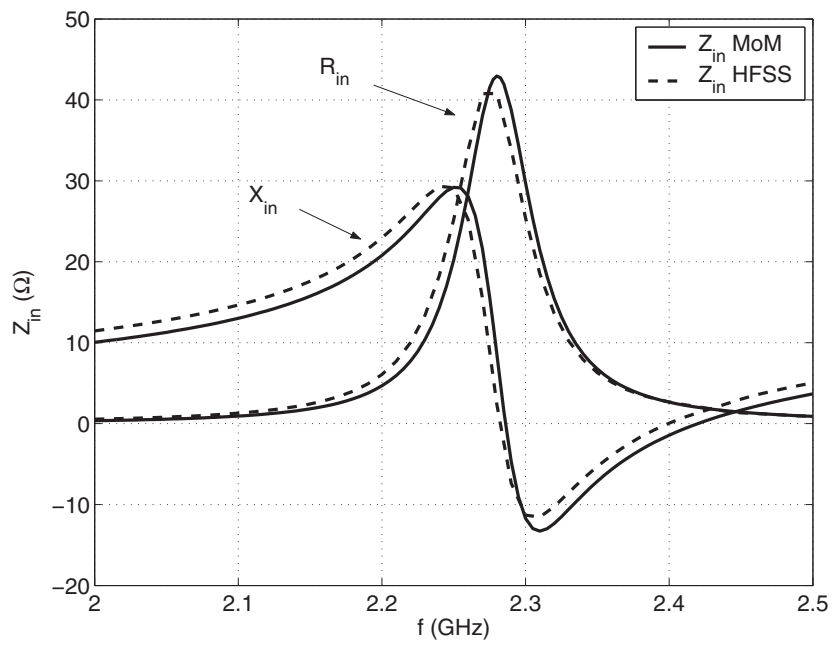

Fig. 9. Input impedance for a cavity-backed wraparound antenna. $b=21 \mathrm{~mm}, a=20 \mathrm{~mm}, z_{1 c}=-25$ $\mathrm{mm}, z_{2 c}=25 \mathrm{~mm}, z_{1 a}=-10 \mathrm{~mm}, z_{2 a}=10 \mathrm{~mm}, z_{f}=5 \mathrm{~mm}, W_{f}=1.45 \mathrm{~mm}, \varepsilon_{r}=9.6,4$ probes at $\phi=$ $0^{\circ}, 90^{\circ}, 180^{\circ}, 270^{\circ}$

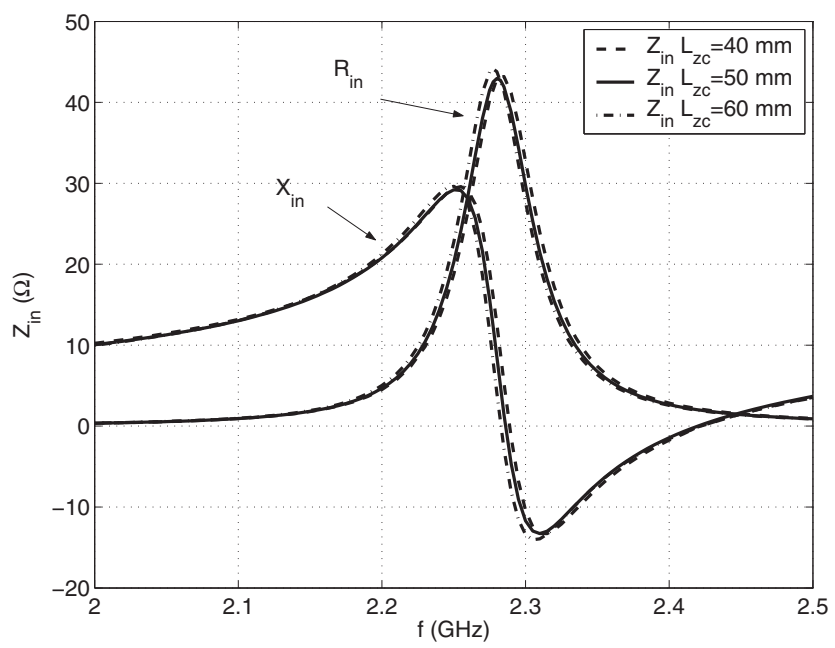

Fig. 10. Input impedance for a cavity-backed wraparound antenna: - - -: $L_{z c}=40 \mathrm{~mm}$, _: $L_{z c}=50 \mathrm{~mm},-.-. . . L_{z c}=60 \mathrm{~mm} . b=21 \mathrm{~mm}, a=20 \mathrm{~mm}, z_{1 a}=-10 \mathrm{~mm}, z_{2 a}=10 \mathrm{~mm}, z_{f}=5 \mathrm{~mm}, W_{f}=1.45$ $\mathrm{mm}, \varepsilon_{r}=9.6,4$ probes at $\phi=0^{\circ}, 90^{\circ}, 180^{\circ}, 270^{\circ}$ 


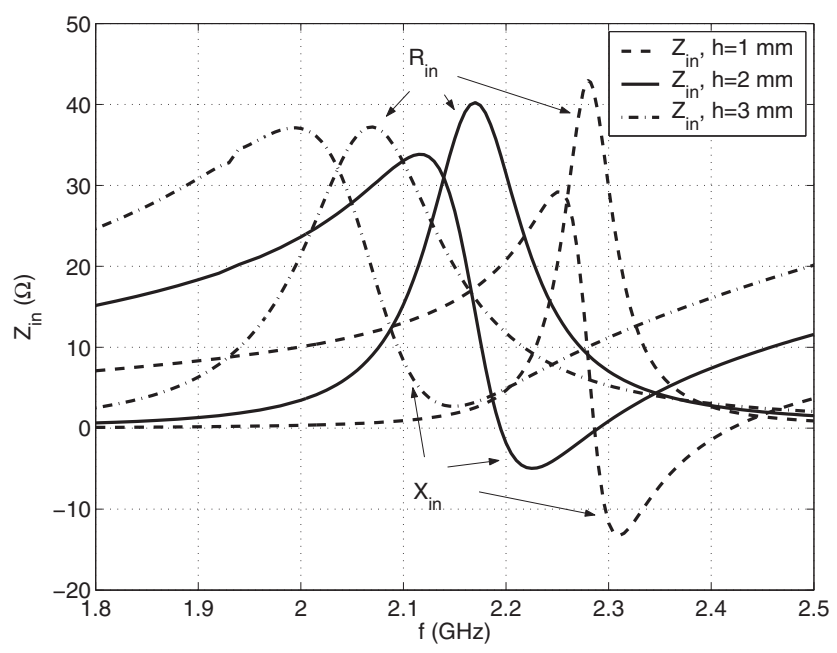

Fig. 11. Input impedance for a cavity-backed wraparound antenna. - - -: $h=1 \mathrm{~mm}, \ldots$ : $h=2$ $\mathrm{mm},-.-.-.: h=3 \mathrm{~mm} . b=21 \mathrm{~mm}, z_{1 c}=-25 \mathrm{~mm}, z_{2 c}=25 \mathrm{~mm}, z_{1 a}=-10 \mathrm{~mm}, z_{2 a}=10 \mathrm{~mm}, z_{f}=5 \mathrm{~mm}$, $W_{f}=1.45 \mathrm{~mm}, \varepsilon_{r}=9.6,4$ probes at $\phi=0^{\circ}, 90^{\circ}, 180^{\circ}, 270^{\circ}$

computational implementation of such solution, and proposes a procedure that allows the analysis for larger cylinders. An approximation for the self-impedance of the probe has also been introduced. The results are validated by comparing to commercial software results. In general it was observed a similar behavior for cavity-backed antenna as for microstrip one. In order to reach an omnidirectional pattern the spacing between probes should be no greater than the dielectric wavelength. The effect of the cavity side wall are limited, and the thickness of the dielectric can be used for obtaining a larger bandwidth.

\section{References}

[Ali et al.,1989] Ali, S.M.; Habashy, T. M.; Kiang, J. F. \& Kong, J. A. (1989). Resonance in cylindrical-rectangular and wraparound structures. IEEE Trans. on Microwave Theory and Techniques, Vol. 37, No. 11, pp. 1773-1783

[Ashkenazy et al.,1985] Ashkenazy, J.; Shrikman, S. \& Treeves, D. (1985). Electric surface current model for the analysis of microstrip antennas on cylindrical bodies, IEEE Trans. on Antennas and Propagation, Vol. 33, No. 3, pp. 295-300

[Erturk \& Rojas,2002] Erturk, V. B. \& Rojas, R. G. (2002). Paraxial space-domain formulation for surface fields on a large dielectric coated circular cylinder. IEEE Trans. on Antennas and Propagation, Vol. 50, No. 11, pp. 1577-87

[Erturk \& Rojas,2003] Erturk, V. B. \& Rojas, R. G. (2003). Efficient analysis of input impedance and mutual coupling of microstrip antennas mounted on large coated cylinders. IEEE Trans. on Antennas and Propagation, Vol. 51, No. 4, pp. 739-749

[Fonseca \& Giarola,1983] Fonseca, S. B. A. \& Giarola A. J. (1983). Analysis of microstrip wraparound antennas using dyadic Greent's function, IEEE Trans. on Antennas and Propagation, Vol. AP-31, No. 2, pp. 248-253 
[Habashy et al.,1990] Habashy, T. M.; Ali, S. M. \& Kong, J. A. (1990). Input impedance and radiation pattern of cylindrical-rectangular and wraparound microstrip antennas, IEEE Trans. on Antennas and Propagation, Vol. 38, No. 5, pp. $722-731$

[Harrington,1961] Harrington, R. F. (1961). Time-harmonic Electromagnetic Fields, McGraw Hill, New York

[Harrington,1968] Harrington, R. F. (1968). Field Computation by Moment Method, IEEE Press, New Jersey

[Heckler et al.,2003] Heckler, M.V.T.; Bonadiman, M., Schildberg, R.; Cividanes, L. \& Lacava, J. C. S. (2003). CAD package to design rectangular probe-fed microstrip antennas conformed on cylindrical structures. Proceedings SBMO/IEEE MTT-S IMOC 2003, pp. $747-752$

[Ke \& Wong,1994] Ke, S. Y. \& Wong, K. L. (1994). Input impedance of a probe-fed superstrate-loaded cylindrical-rectangular microstrip antenna. Microwave and Optical Technology Letters, Vol, 7, No. 5, pp. 232-36

[Kempel \& Volakis,1995] Kempel L. C. \& Volakis, J. L. (1995). Radiation by cavity-backed antennas on a circular cylinder, IEE Proceedings on Microwaves, Antennas $\mathcal{E}$ Propagation, Vol. 142, No. 3, pp. 233-239

[Krowne,1983] Krowne, C.C. (1983). Cylindrical-rectangular microstrip antennas. IEEE Trans. on Antennas and Propagation, Vol. 31, No. 1, pp. 194-199

[Luk et al.,1989] Luk, K. M.; Uk, K.M.; Lee K. F. \& Dahele J. S. (1989). Analysis of the cylindrical-rectangular microstrip patch antenna. IEEE Trans. on Antennas and Propagation, Vol. 37, No. 2, pp. 143-147

[Lumini,1991] Lumini, F. (1991). Analysis and design of rectangular microstrip antennas over cylindrical surfaces. MsC. Thesis, Technological Institute of Aeronautics, (in portuguese)

[Mang \& Xiaowen,2004] Mang, H. \& Xiaowen, X. (2004). Full-wave analysis and wide-band design of probe-fed multilayered cylindrical-rectangular microstrip antennas. IEEE Trans. on Antennas and Propagation, Vol. 52, No. 7, pp. 1749-57

[Munson,1974] Munson, R. E. (1974). Conformal microstrip antennas and microstrip phased arrays. IEEE Trans. on Antennas and Propagation, Vol. 22, No. 1, pp. 74-78

[Pereira Filho,2009] Pereira Filho, O. M. C. (2009) Flush-mounted cylindrical-rectangular microstrip antennas. IET Microwaves, Antennas \& Propagation, Vol. 3, No. 1, pp. 1-13

[Raffaelli et al.,2005] Raffaelli, S.; Sipus, Z. \& Kildal, P. S. (2005). Analysis and measurements of conformal patch array antennas on multilayer circular cylinder. IEEE Trans. on Antennas and Propagation, Vol. 53, No. 3, pp. 1105-13

[Silva et al.,1991] Silva, C. M.; Lumini, F.; Lacava, J. C. S. \& Richards, F. P. (1991). Analysis of cylindrical arrays of microstrip rectangular patches. Electronic Letters, Vol. 27, No. 9, pp. $778-780$

[Silva \& Lacava,1995] Silva, C. M. \& Lacava, J. C. S. (1995). Mutual impedance of conformal cylindrical microstrip antenna arrays with a protection layer. Proceedings 1995 SBMO/IEEE MTT-S International Conference, pp. 314-319

[Silva et al.,1991b] Silva, F. C.; Fonseca, S. B. A.; Soares, A. J. M. \& Giarola A. J. (1991). Analysis of microstrip antennas on circular-cylindrical substrates with a dielectric overlay. IEEE Trans. on Antennas and Propagation, Vol. 39, No. 9, pp. 1398-1403 
[Tam et al.,1995] Tam W. Y.; Lai, A. K. Y. \& Luk, K. M. (1995). Cylindrical rectangular microstrip antennas with coplanar parasitic patches, IEE Proc. on Microwave, Antennas and Propagation, Vol. 142, No. 4, pp. 300-306

[Tulintseff et. al.,1991] Tulintseff, A. N.; Al, S. M. \& Kong, J. A. (1991). Input impedance of a probe-fed stacked circular microstrip antenna. IEEE Trans. on Antennas and Propagation, Vol. 39, No. 3, pp. 381-390

[Wong \& Ke,1993] Wong, K. L. \& Ke, S. Y. (1993). Characteristics of the cylindrical wraparound microstrip patch antenna, roc. Natl. Sci. Counc. ROC(A), Vol. 17, No. 6, pp. $438-42$

[Wong et al.,1993b] Wong, K. L.; Cheng, Y. T. \& Row, J. S. (1993). Resonance in a superstrate-loaded cylindrical-rectangular microstrip structure. IEEE Trans. on Microwave Theory and Techniques, Vol. 41, No. 5, pp. 814-819

[Wong,1999] Wong, K. L. (1999). Design of Nonplanar Microstrip Antennas and Transmission Lines, Wiley-Interscience

[Yang \& Ruan,1993] Yang, C. \& Ruan, Y.Z. (1993). Radiation characteristics of wraparound microstrip antenna on cylindrical body. Electronic Letters, Vol. 29, No. 6, pp. 512-14

[Zheng \& Chang,1991] Zheng, J. X. \& Chang, D. C. (1991). End-correction network of a coaxial probe for microstrip patch antennas. IEEE Trans. on Antennas and Propagation, Vol. 39, No. 1, pp. 115-118 


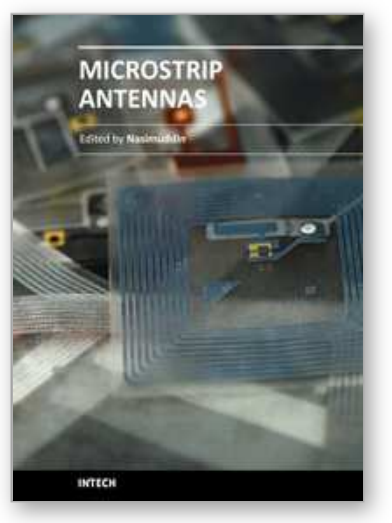

\author{
Microstrip Antennas \\ Edited by Prof. Nasimuddin Nasimuddin
}

ISBN 978-953-307-247-0

Hard cover, 540 pages

Publisher InTech

Published online 04, April, 2011

Published in print edition April, 2011

In the last 40 years, the microstrip antenna has been developed for many communication systems such as radars, sensors, wireless, satellite, broadcasting, ultra-wideband, radio frequency identifications (RFIDs), reader devices etc. The progress in modern wireless communication systems has dramatically increased the demand for microstrip antennas. In this book some recent advances in microstrip antennas are presented.

\title{
How to reference
}

In order to correctly reference this scholarly work, feel free to copy and paste the following:

O. M. C. Pereira-Filho, T. B. Ventura, C. G. Rego, A. F. Tinoco-S., and J. C. da S. Lacava (2011). CavityBacked Cylindrical Wraparound Antennas, Microstrip Antennas, Prof. Nasimuddin Nasimuddin (Ed.), ISBN: 978-953-307-247-0, InTech, Available from: http://www.intechopen.com/books/microstrip-antennas/cavitybacked-cylindrical-wraparound-antennas

\section{INTECH}

open science | open minds

\author{
InTech Europe \\ University Campus STeP Ri \\ Slavka Krautzeka 83/A \\ 51000 Rijeka, Croatia \\ Phone: +385 (51) 770447 \\ Fax: +385 (51) 686166 \\ www.intechopen.com
}

\author{
InTech China \\ Unit 405, Office Block, Hotel Equatorial Shanghai \\ No.65, Yan An Road (West), Shanghai, 200040, China \\ 中国上海市延安西路65号上海国际贵都大饭店办公楼 405 单元 \\ Phone: +86-21-62489820 \\ Fax: +86-21-62489821
}


(C) 2011 The Author(s). Licensee IntechOpen. This chapter is distributed under the terms of the Creative Commons Attribution-NonCommercialShareAlike-3.0 License, which permits use, distribution and reproduction for non-commercial purposes, provided the original is properly cited and derivative works building on this content are distributed under the same license. 